TP Periodica Polytechnica

Mechanical Engineering

60(1), pp. 41-49, 2016

DOI: $10.3311 /$ PPme. 8518

Creative Commons Attribution (i)

RESEARCH ARTICLE

\section{Validation of a Discrete Model for Flow Distribution in Dividing-Flow Manifolds: Numerical and Experimental Studies}

\author{
András Tomor ${ }^{1 *}$, Gergely Kristóf ${ }^{1}$
}

Received 26 August 2015; accepted after revision 08 December 2015

\begin{abstract}
A novel discrete model with variable flow coefficients is developed for dividing-flow manifold design. The applicability and accuracy of the new model is also investigated, and a validation procedure is performed. Dimensionless volume flow rate distributions along a simple dividing-flow manifold are calculated with two different approaches: the discrete model and a three-dimensional CFD model are applied. In order to validate the calculated results, laboratory experiments are carried out. Results of the discrete model compare favourably with high resolution CFD results and also with experimental data, which underlines the applicability of the new loss coefficient parametrisation used in the discrete model.
\end{abstract}

\section{Keywords}

$C F D$, discrete model, dividing-flow manifold, experimental validation, flow distribution

\section{Introduction}

Flow in manifolds is an essential part of several technical processes in which a large fluid stream is to be divided into more parallel streams, the streams are to be operated separately and then collected into one stream. For example, flow manifolds are used in air engineering [1], water and wastewater treatment [2], polymer processing [3], and they are also important elements of chemical reactors [4], fuel cells [5, 6] and heat exchangers [7]. Manifolds can be classified into the following types: combining-, dividing-, reverse-, and parallel- flow manifolds as can be seen in Fig. 1.

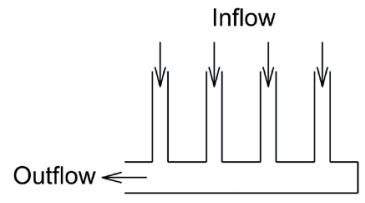

(a) Combining

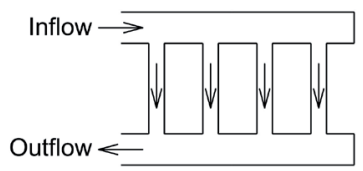

(c) Reverse

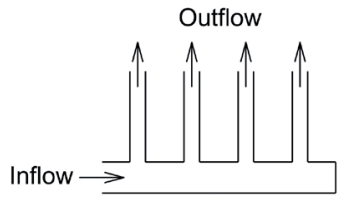

(b) Dividing

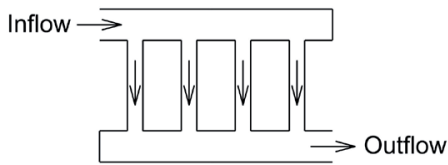

(d) Parallel
Fig. 1 Different types of flow manifolds

Due to the large number of applications, several studies focus on the investigation of flow manifolds and construct models for manifold design (see a recent review by Hassan et al. [8]. Achieving uniform flow distribution is usually the primary goal of a manifold system design; the uniformity often determines the efficiency of the technical process and the durability of applied devices.

There are four approaches to investigate flow distribution and pressure drop in a flow manifold: analytical models [4-7], computational fluid dynamics (CFD) models [9-16], discrete models [17-19] and experimental measurements [11, 14, 20-22].

In an analytical (or continuous) model, the flow branching off is considered to be continuous along the manifold. 
Continuous models solve sets of ordinary differential equations, and the solutions are usually fully explicit. Due to the compact and simple solutions, analytical models are flexible and simple for designers. However, continuous manifolds are limiting cases of discrete manifolds [23].

In discrete models, manifolds are regarded as networks of several junctions. A discrete model solves a system of difference equations by means of an iterative method, and the designer usually needs to use a computer programme for the calculations. This approach can be expansively applicable for design tasks if the flow coefficients, such as the pressure recovery, turning loss and friction coefficients, are accurately determined.

CFD is a modern approach; it is continuously developing and increasingly popular among researchers. A CFD model can resolve real engineering structures and provide detailed pressure and flow field results. The major advantage of this approach over the two earlier methods is that the flow coefficients (loss coefficients) are not needed. However, the reliability of these results is often questionable. Moreover, CFD for optimizing complete manifold geometries has high computational cost - particularly for 3D complex structures.

Results of high-standard experiments can be used for the validation of various model results. Reference data from reliable experiments are valuable per se. Nevertheless, these investigations are usually time-consuming and the applied devices are often expensive. Most of the available experimental investigations [2022] contain old experimental data without specified error bars; therefore, it is not appropriate to use them as reference data.

In the present paper, a novel discrete model for dividingflow manifold design is formulated. Instead of applying constant pressure recovery, turning loss and friction coefficients, variable flow coefficients are implemented in the model. Wang and Wang [19] use also varying coefficients; however, in the present model, a recently developed correlation for the coefficient of turning losses [24] is involved and tested.

Flow distributions along a simple dividing-flow manifold are determined by means of three different approaches consisting of two theoretical models and laboratory measurements. The applicability and accuracy of the discrete model including the mentioned loss coefficient formulation is investigated by comparing its calculated results with own experimental data. A CFD model is also constructed, and a thorough validation procedure is performed.

\section{Models for flow distribution in a dividing-flow manifold}

In our study, a dividing-flow manifold with five ports and a specified geometry is investigated (Fig. 2). The inner diameters of the header and ports are $D_{1}=20 \mathrm{~mm}$ and $D_{2}$ $=10 \mathrm{~m}$, respectively. The length of each branch tube is $L_{2}$ $=12.5 \mathrm{~mm}$, and the distance of two neighbouring laterals is $L_{1}=60 \mathrm{~mm}$. Dimensionless volume flow rate $\left(q_{v 2}(i) / q_{v 0}\right)$ distributions along the manifold are calculated with two different approaches: a new discrete model and a CFD based approach are applied. In order to validate the results, laboratory experiments are also carried out.

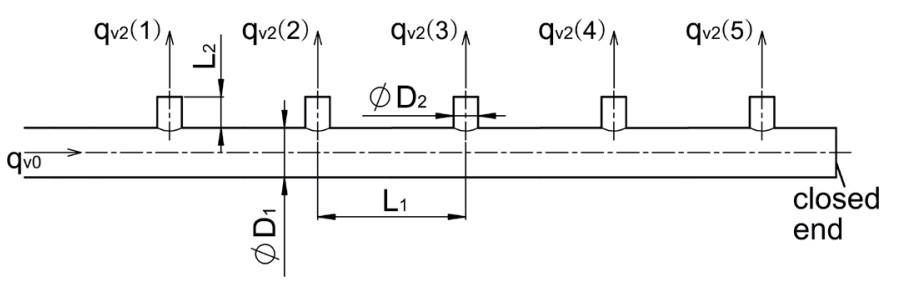

Fig. 2 Geometrical model of the investigated dividing-flow manifold

\subsection{Discrete model}

In the present paper, steady-state incompressible singlephase flow is considered under isothermal conditions. Before the first lateral, fully developed flow is assumed. The schematic of the discrete model of the dividing-flow manifold can be seen in Fig. 3. The control volumes for the $i$ th section are also indicated.

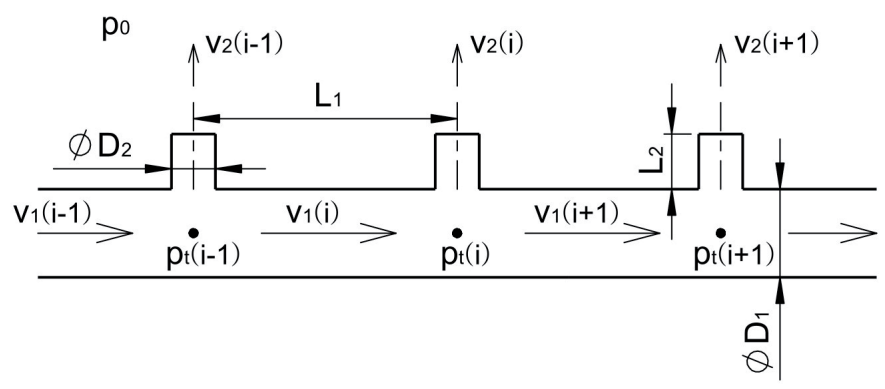

(a)

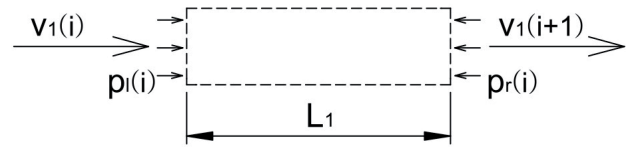

b)

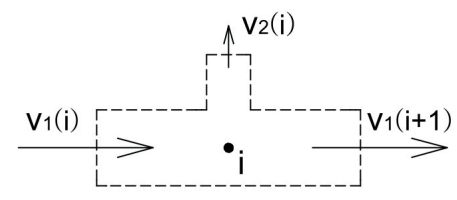

c)

Fig. 3 Schematic of the discrete model (a) along with the control volume for momentum in axial direction (b) and control volume for continuity (c)

The parameters of the system are the Reynolds number at the manifold inlet $\left(R e_{0}\right)$, the diameter ratio $\left(D_{2} / D_{1}\right)$, the ratio of port length to port diameter $\left(L_{2} / D_{2}\right)$, the ratio of the distance between two ports to header diameter $\left(L_{1} / D_{1}\right)$ and the total number of ports $(n)$. The actual control parameter is the Reynolds number at the manifold inlet.

Considering the control volume for momentum in Fig. 3, the momentum equation in the axial direction can be written as 


$$
p_{l}(i)-p_{r}(i)=\Delta P_{f}+k(i) \rho\left(v_{1}^{2}(i+1)-v_{1}^{2}(i)\right),
$$

where $p_{l}$ and $p_{r}$ are upstream and downstream static pressures, respectively; $\Delta P_{f}$ is the friction loss, $v_{1}$ is the flow velocity in the header, $\rho$ is the fluid density, $k$ is the pressure recovery factor and $i=1,2, \ldots n$, where $n$ denotes the total number of laterals. In our case, $n=5$. In the course of the branching process, some of the fluid entering the port retains a part of its initial axial momentum as it crosses the control surface and leaves the header [25]. This momentum recovery phenomenon is taken into consideration by introducing an empirical coefficient $k$ in the momentum equation.

A modified Bernoulli equation between $p_{t}(i-1)$ and $p_{t}(i)$ can be obtained by choosing the appropriate location of the control volume for momentum (in an extreme case, the control surfaces are at the junctions), using that

$$
\begin{aligned}
& p_{t}(i-1) \cong p_{l}(i)+\frac{\rho}{2} v_{1}^{2}(i) \quad \text { and } \\
& p_{t}(i) \cong p_{r}(i)+\frac{\rho}{2} v_{1}^{2}(i+1),
\end{aligned}
$$

and modifying Eq. (1) as follows:

$$
\begin{aligned}
& p_{t}(i-1)-p_{t}(i)= \\
& \quad \lambda_{1}(i) \frac{L_{1}}{D_{1}} \frac{\rho}{2} v_{1}^{2}(i)+(1-2 k(i))\left(\frac{\rho}{2} v_{1}^{2}(i)-\frac{\rho}{2} v_{1}^{2}(i+1)\right)
\end{aligned}
$$

where $p_{t}$ is the total pressure and $\lambda_{1}$ is the pipe friction factor for the header flow. Wang et al. [4] provide an expansively applicable formula for the calculation of the pressure recovery factor $k(i)$ :

$$
k(i)=\alpha+\beta \frac{v_{1}^{2}(i)-v_{1}^{2}(i+1)}{v_{1}^{2}(i)},
$$

in which $\alpha$ and $\beta$ are constants depend only on the manifold geometry. For the present geometry, $\alpha=0.5$ and $\beta=0.1$ [4].

The Bernoulli equation for the port flow can be written as follows:

$$
\begin{aligned}
p_{t}(i)-p_{0} & = \\
& \left(1+C_{f}(i)+\lambda_{2}(i) \frac{L_{2}}{D_{2}}\right) \frac{\rho}{2} v_{2}^{2}(i)=\left(1+C_{f e}(i)\right) \frac{\rho}{2} v_{2}^{2}(i),
\end{aligned}
$$

in which $C_{f}$ is the turning loss coefficient, $\lambda_{2}$ is the pipe friction factor for the lateral flow, $v_{2}$ is the flow velocity in the port and $C_{f e}$ is the extended turning loss coefficient.

The extended turning loss coefficient, $C_{f e}(i)$, can be calculated according to the following formulation introduced by Kristóf and Tomor [24]:

$$
C_{f e}(i)=\left\{\begin{array}{cl}
\operatorname{CRe}_{1}^{D}(i)+\left(\frac{v_{2}(i)}{v_{1}(i)}\right)^{-2}, & \text { if } \frac{v_{2}(i)}{v_{1}(i)} \leq C V R(i) \\
A_{2} \operatorname{Re}_{1}^{B_{2}}(i)+A_{1} \operatorname{Re}_{1}^{B_{1}}(i)\left(\frac{v_{2}(i)}{v_{1}(i)}\right)^{-2}, & \text { if } \frac{v_{2}(i)}{v_{1}(i)}>\operatorname{CVR}(i)
\end{array},\right.
$$

where $R e_{1}$ is the Reynolds number in the header, $A_{1}, A_{2}, B_{1}, B_{2}$, $C$ and $D$ are constants depend on the geometry and the critical velocity ratio, $C V R(i)$, can be calculated as

$$
C V R(i)=\sqrt{\frac{A_{1} \operatorname{Re}_{1}^{B_{1}}(i)-1}{C \operatorname{Re}_{1}^{D}(i)-A_{2} \operatorname{Re}_{1}^{B_{2}}(i)}} .
$$

For the present geometry, $A_{1}=5.143, A_{2}=0.271, B_{1}=-0.099$, $B_{2}=0.080, C=0.434$ and $D=0.058$ as can be seen in Table 1 . The method and calculations for the determination of the constants in Table 1 are described in the previous study of Kristóf and Tomor [24]. The loss coefficient formulation is elaborated using validated results of modern three-dimensional computational fluid dynamics methods. The loss coefficients are determined by a nonlinear fit, and the constants are obtained by the fitting procedure.

The value of $C_{f e}(i)$ also involves the friction loss due to the port wall friction; therefore, calculation of $\lambda_{2}(i)$ is not required in the present model. For hydraulically smooth pipes, the following well-known formulas [4] can be used for the calculation of the $\lambda_{1}(i)$ friction factor:

$$
\begin{gathered}
\lambda_{1}(i)=\frac{64}{\operatorname{Re}_{1}(i)} \text { for } \operatorname{Re}_{1}(i)<2200, \\
\lambda_{1}(i)=\frac{0.3164}{\operatorname{Re}_{1}^{0.25}(i)} \text { for } 2200 \leq \operatorname{Re}_{1}(i) \leq 10^{5} \text { and, } \\
\lambda_{1}(i)=0.0032+\left(\frac{0.221}{\operatorname{Re}_{1}^{0.237}(i)}\right) \text { for } \operatorname{Re}_{1}(i)>10^{5},
\end{gathered}
$$

The continuity equation is

$$
\frac{D_{1}^{2} \pi}{4}\left(v_{1}(i)-v_{1}(i+1)\right)=\frac{D_{2}^{2} \pi}{4} v_{2}(i)
$$

The boundary conditions can be specified as

$$
\begin{gathered}
v_{1}(1)=v_{0} \text { and } \\
v_{1}(n+1)=0 .
\end{gathered}
$$

Flow distributions are calculated for three different Reynolds numbers at the inlet (13200, 26000 and 39200), and accordingly the $v_{0}$ values are set to $10.20 \mathrm{~m} / \mathrm{s}, 20.05 \mathrm{~m} / \mathrm{s}$ and $30.25 \mathrm{~m} / \mathrm{s}$, respectively.

The formulation of the hydraulic network model is represented by Eqs. (4)-(14); the system of equations can be solved iteratively, e.g., using the Newton-Raphson method. 
Table 1 Constants for the calculation of the extended turning loss coefficient

\begin{tabular}{|c|c|c|c|c|c|c|c|}
\hline$D_{2} / D_{1}[-]$ & $L_{2} / D_{1}[-]$ & $A_{1}[-]$ & $B 1[-]$ & $A_{2}[-]$ & $B 2[-]$ & $C[-]$ & $D[-]$ \\
\hline 0.2 & 0.1 & 10.083 & -0.114 & 0.873 & 0.031 & 0.939 & 0.031 \\
\hline 0.3 & 0.1 & 5.880 & -0.087 & 0.860 & 0.031 & 0.950 & 0.031 \\
\hline 0.4 & 0.1 & 4.204 & -0.071 & 0.827 & 0.029 & 0.942 & 0.030 \\
\hline 0.5 & 0.1 & 3.617 & -0.068 & 0.798 & 0.030 & 0.964 & 0.029 \\
\hline 0.625 & 0.1 & 3.480 & -0.075 & 0.554 & 0.063 & 0.793 & 0.048 \\
\hline 0.75 & 0.1 & 3.513 & -0.084 & 0.344 & 0.105 & 0.646 & 0.068 \\
\hline 0.875 & 0.1 & 2.832 & -0.071 & 0.322 & 0.112 & 0.629 & 0.073 \\
\hline 1 & 0.1 & 2.211 & -0.055 & 0.386 & 0.099 & 0.774 & 0.057 \\
\hline 0.2 & 0.3 & 3.633 & -0.045 & 0.828 & 0.009 & 0.832 & 0.013 \\
\hline 0.3 & 0.3 & 5.871 & -0.087 & 0.612 & 0.036 & 0.691 & 0.035 \\
\hline 0.4 & 0.3 & 7.123 & -0.111 & 0.374 & 0.080 & 0.535 & 0.064 \\
\hline 0.5 & 0.3 & 4.435 & -0.083 & 0.401 & 0.075 & 0.551 & 0.065 \\
\hline 0.625 & 0.3 & 5.677 & -0.114 & 0.171 & 0.150 & 0.377 & 0.101 \\
\hline 0.75 & 0.3 & 4.299 & -0.099 & 0.114 & 0.190 & 0.313 & 0.123 \\
\hline 0.875 & 0.3 & 2.739 & -0.068 & 0.201 & 0.151 & 0.437 & 0.102 \\
\hline 1 & 0.3 & 2.863 & -0.078 & 0.184 & 0.176 & 0.481 & 0.109 \\
\hline 0.2 & 0.625 & 12.968 & -0.168 & 0.643 & 0.006 & 0.737 & -0.002 \\
\hline 0.3 & 0.625 & 5.377 & -0.093 & 0.557 & 0.018 & 0.635 & 0.015 \\
\hline 0.4 & 0.625 & 5.147 & -0.092 & 0.423 & 0.042 & 0.553 & 0.033 \\
\hline 0.5 & 0.625 & 5.143 & -0.099 & 0.271 & 0.080 & 0.434 & 0.058 \\
\hline 0.625 & 0.625 & 6.079 & -0.124 & 0.094 & 0.176 & 0.169 & 0.149 \\
\hline 0.75 & 0.625 & 3.855 & -0.091 & 0.136 & 0.142 & 0.353 & 0.083 \\
\hline 0.875 & 0.625 & 2.352 & -0.056 & 0.249 & 0.099 & 0.464 & 0.067 \\
\hline 1 & 0.625 & 2.356 & -0.064 & 0.167 & 0.157 & 0.445 & 0.088 \\
\hline 0.2 & 1.25 & 23.848 & -0.224 & 0.926 & -0.029 & 1.116 & -0.041 \\
\hline 0.3 & 1.25 & 6.257 & -0.125 & 0.759 & -0.017 & 0.903 & -0.027 \\
\hline 0.4 & 1.25 & 3.292 & -0.074 & 0.661 & -0.010 & 0.777 & -0.016 \\
\hline 0.5 & 1.25 & 2.718 & -0.059 & 0.526 & 0.003 & 0.635 & 0 \\
\hline 0.625 & 1.25 & 2.781 & -0.062 & 0.338 & 0.033 & 0.501 & 0.019 \\
\hline 0.75 & 1.25 & 2.652 & -0.062 & 0.200 & 0.074 & 0.390 & 0.042 \\
\hline 0.875 & 1.25 & 2.688 & -0.067 & 0.162 & 0.090 & 0.419 & 0.037 \\
\hline 1 & 1.25 & 2.069 & -0.051 & 0.150 & 0.110 & 0.379 & 0.054 \\
\hline 0.2 & 2 & 42.031 & -0.265 & 1.291 & -0.051 & 1.626 & -0.067 \\
\hline 0.3 & 2 & 9.734 & -0.165 & 0.987 & -0.037 & 1.247 & -0.053 \\
\hline 0.4 & 2 & 3.770 & -0.095 & 0.885 & -0.035 & 1.109 & -0.048 \\
\hline 0.5 & 2 & 2.234 & -0.051 & 0.533 & -0.041 & 0.677 & -0.047 \\
\hline 0.625 & 2 & 1.585 & -0.025 & 0.892 & -0.054 & 0.932 & -0.046 \\
\hline 0.75 & 2 & 1.666 & -0.028 & 0.469 & -0.028 & 0.597 & -0.036 \\
\hline 0.875 & 2 & 1.981 & -0.044 & 0.209 & 0.024 & 0.530 & -0.022 \\
\hline 1 & 2 & 1.286 & -0.018 & 0.325 & 0.010 & 0.641 & -0.032 \\
\hline
\end{tabular}




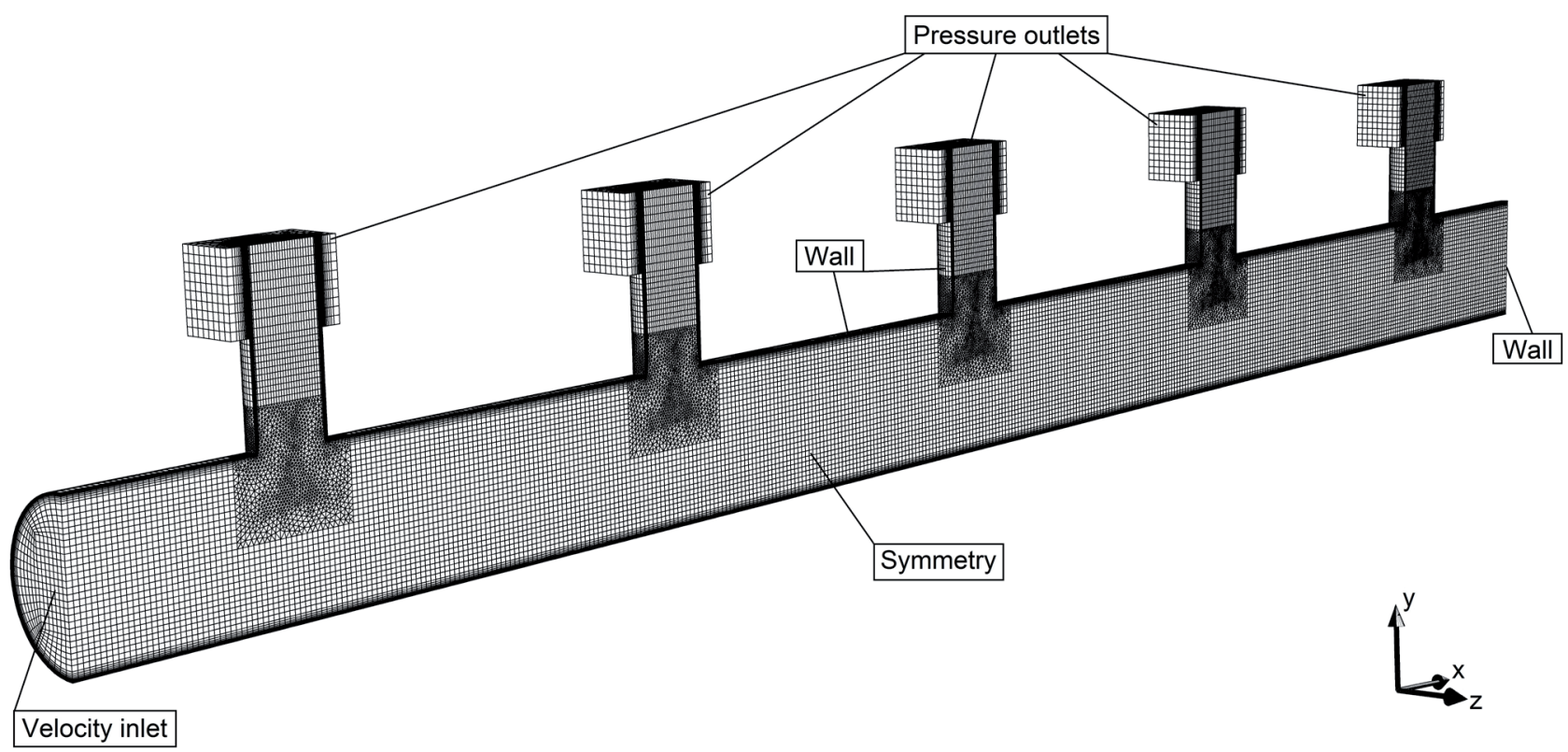

Fig. 4 The numerical mesh for the investigated manifold and the boundary conditions

\subsection{CFD model}

A slightly simplified manifold geometry is created by ANSYS DesignModeler, and the numerical mesh is prepared using ANSYS Meshing in ANSYS Workbench 14.5. Due to the symmetry, it is sufficient to model only one half of the manifold.

The MultiZone method of ANSYS Meshing allows us to construct high-quality meshes. The quality of these grids is similar to that of the well-constructed block-structured grids. In the vicinity of the ports, some tetrahedral elements are used instead of the dominant hexahedral cells. Near the walls, an inflation layer is generated applying prismatic elements.

The simulations are executed using ANSYS FLUENT 14.5. The model solves the standard three-dimensional Reynoldsaveraged Navier-Stokes and continuity equations for steady state turbulent flow based on the $k-\omega$ SST turbulence model [26]. Some previous studies $[11,16]$ use the standard $k-\varepsilon$ turbulence model; however, the boundary layer is greatly resolved in the present study, and the $k-\omega$ SST turbulence model is more feasible in our case [26]. The pressure based solver is applied, and the working fluid is incompressible air. The Coupled solution algorithm is used for the pressurevelocity coupling. Every flux formulation is implemented using the second order upwind differencing scheme.

At the inlet of the header, velocity inlet boundary condition is prescribed: The profiles for turbulent quantities and velocity are defined from the results of auxiliary simulations of fully developed flow in an infinite length cylindrical pipe modelled with the help of periodic inlet-outlet conditions. Due to the relatively short port lengths, the presence of recirculating flows is expected at the outlets of ports; therefore, pressure outlet boundary conditions are applied on the boundaries of rectangular boxes attached to the port outlets in order to achieve realistic outlet conditions. Here, the pressure is set to zero. Symmetry and wall boundary conditions are prescribed according to the plane of symmetry and pipe wall, respectively. At the closed end of the header, wall boundary condition is applied. The numerical mesh for the investigated manifold with the prescribed boundary conditions is shown in Fig. 4.

In the course of the simulations, the iterations are carried on until iterative convergence is achieved with at least four orders of magnitude decrease in every residual. The results are also checked for mesh independence, and a mesh with a cell number of about 1,200,000 is accepted. This mesh is refined twice successively by a refinement factor of 1.5 . The uncertainty due to discretization is estimated based on the Grid Convergence Index (GCI) method [27]. The uncertainty estimation is done for local velocities and pressure values, and all the estimated relative errors are below $3.5 \%$. Simulations are run for three different Reynolds numbers at the inlet (13200, 26000 and 39200). A representative result of the velocity distributions can be seen in Fig. 5 for $R e_{0}=39200$.
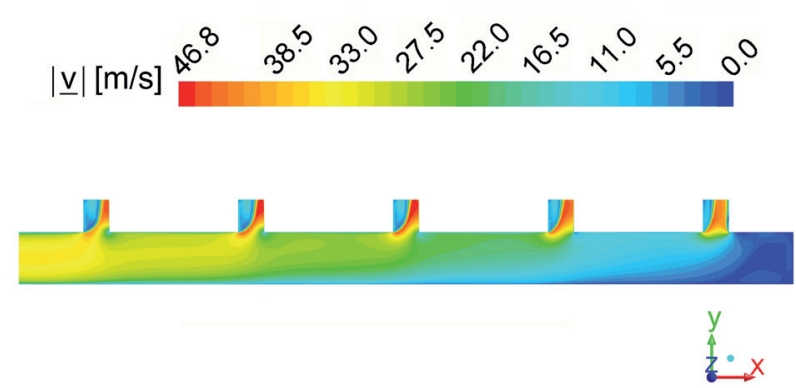

Fig. 5 Velocity distribution in the plane of symmetry of the ivestigated manifold - contours of velocity magnitude. $R e_{0}=39200$. 


\subsection{Physical model and experimental setup}

A simple experimental system including a dividing-flow manifold with the previously presented geometry (see Fig. 2) is constructed, and dimensionless volume flow rate distributions along the manifold are determined for three different Reynolds numbers at the manifold inlet. In the course of the measurements, the working fluid is air with a constant temperature of $24^{\circ} \mathrm{C}$, and the ambient pressure is $101 \mathrm{kPa}$. The experimental setup and concept are the following (Fig. 6):

The flow is driven by a blower with adjustable rotational speed, which is connected to a hydraulically smooth PVC pipe with an inner diameter of $D_{0}=59.4 \mathrm{~mm}$. A standardized [28] through-flow orifice plate is built into the pipe in order to measure the reference volume flow rate. A confuser is connected to the open end of the PVC pipe, and the manifold is placed at the confuser outlet. The manifold is fabricated from a hydraulically smooth aluminium pipe with an inner diameter of $D_{1}=20 \mathrm{~mm}$. The distance between the first port of the manifold and the confuser outlet is $4.5 D_{1}$. Volume flow rates through ports are deduced from velocity data measured by a small-diameter $(0.6 \mathrm{~mm})$ Pitot probe.

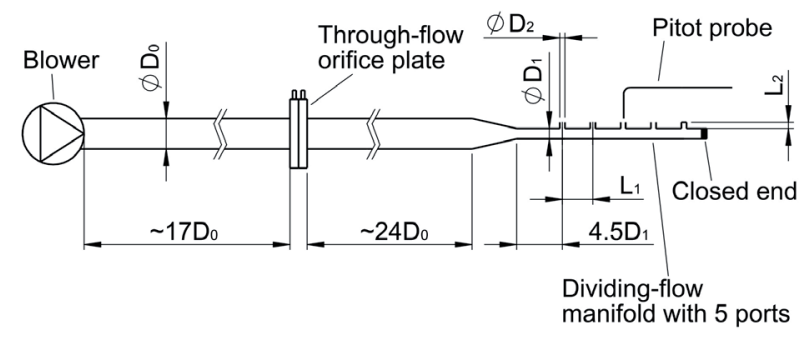

Fig. 6 Schematic of the experimental setup

As can be seen in Fig. 5, the flow is not fully developed at any of the port outlets; in this case, the streamlines are not parallel. However, in the jets that leave the ports, the following equality is assumed to be valid considering the ambient pressure the reference $\left(p_{0}=0\right)$ :

$$
p_{t p}=\frac{\rho}{2} v_{p}^{2},
$$

where $p_{t p}$ is the total pressure measured by means of the Pitot probe and $v_{p}$ is the flow velocity at the measurement point. This assumption can lead to small distortions of the measured velocity values and the determined volume flow rates; therefore, it slightly increases the experimental uncertainty.

Figure 7(a) shows a typical result for velocity distribution in the outlet cross section of a port obtained from CFD simulations. In this situation, it is inadvisable to use standard measurement techniques, such as the log-lin method [29]. Hence, a unique method consisting of 21 measurement points is applied in order to achieve a sufficiently high resolution in the investigated cross section and to keep the measurement time to a practicable minimum. The location of the 21 measurement points can be seen in Fig. 7(b); these points are located on a plane at a distance of $3 \mathrm{~mm}$ from the port outlet.

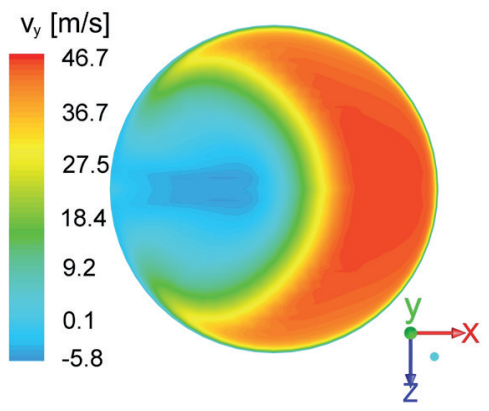

(a)

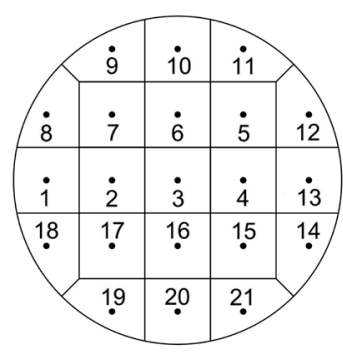

(b)
Fig. 7 A typical result for velocity distribution in the outlet cross section of a port (third port, $R e_{0}=39200$ ) (a) and the location of the 21 measurement points (b)

Volume flow rate through the $i$ th port can be calculated as

$$
q_{v 2}(i)=\sum_{j=1}^{21} v_{p}(i, j) A_{p}(i, j),
$$

where $A_{p}$ is the area that belongs to the $j$ th measurement point of the $i$ th port.

The intrusiveness of the probe can be reduced by choosing the mentioned $3 \mathrm{~mm}$ distance instead of measuring directly in the outlet cross section. Moreover, negative $v_{v}$ velocities are negligible at this distance according to our CFD results, and the introduced measurement method is applicable. However, volume flow rate in the free jet increases with the increase of the distance from the outlet due to the entrainment, which causes a bias error in volume flow rate measurements.

Experimental uncertainty of volume flow rates through ports consists of more error types. Pressure differences are measured with calibrated digital manometers with a measurement accuracy of $\pm 2 \mathrm{~Pa}$; considering this accuracy, precision errors of the determined volume flow rates are calculated according to the propagation of error [30]. The applied resolution consisting of 21 measurement points involves a small uncertainty per se, which is estimated by means of CFD simulations, together with the previously presented bias error caused by measuring at a distance of $3 \mathrm{~mm}$ from the port outlet. Volume flow rates are reported directly, and they are also deduced from velocity data according to a virtual measurement. Results of the two different methods are compared to each other, and accordingly the error can be estimated. From the CFD results, confidence interval estimation with 95 percent confidence is performed assuming normal distribution. Uncertainty that arises from using Eq. (15) is several orders of magnitude smaller than other errors and can be neglected. The total experimental uncertainty is the square root of sum of squares of the different errors [30]. It is important to note that uncertainties of dimensionless volume flow rates are estimated to be smaller than those of non-dimensionless volume flow 
rates due to the similarity of velocity distributions in the outlet cross sections of ports, and they will be reported later.

Reference volume flow rates measured by the orifice plate also involve experimental uncertainty, which is estimated according to the standard [28]. It is important to emphasize that the present configuration (see Fig. 6) involves $0.5 \%$ additional uncertainty. Moreover, the total uncertainty of the reference volume flow rate measurements includes the precision error that is calculated from the measurement accuracy $( \pm 2 \mathrm{~Pa})$ of the calibrated manometers according to the propagation of error [30].

The three different volume flow rates measured by the through-flow orifice plate are $0.0032 \pm 0.0001 \mathrm{~m}^{3} / \mathrm{s}$, $0.0063 \pm 0.0002 \mathrm{~m}^{3} / \mathrm{s}$ and $0.0095 \pm 0.0003 \mathrm{~m}^{3} / \mathrm{s}$, and accordingly the three Reynolds numbers are $13200 \pm 400,26000 \pm 800$ and $39200 \pm 1200$, respectively.

\section{Results and discussion}

Dimensionless volume flow rate distributions along the investigated dividing-flow manifold are determined by three different methods, and results of the different approaches are compared to each other. In order to obtain dimensionless quantities, volume flow rates through ports are divided by the total volume flow rate, $q_{v 0}$, which is the sum of the branched off volume flow rates and belongs to $R e_{0}$. Results for three different Reynolds numbers at the manifold inlet are summarized in Table 2, and some representative distributions are illustrated in Fig. 8.

Table 2 Dimensionless volume flow rates through ports for three different Reynolds numbers at the manifold inlet

\begin{tabular}{|c|c|c|c|c|}
\hline$R e_{0}$ & $\begin{array}{l}\text { No. of } \\
\text { ports }\end{array}$ & CFD & $\begin{array}{l}\text { Discrete } \\
\text { model }\end{array}$ & Experiment \\
\hline \multirow{5}{*}{13200} & 1 & 0.157 & 0.155 & $0.159 \pm 0.006$ \\
\hline & 2 & 0.177 & 0.179 & $0.178 \pm 0.006$ \\
\hline & 3 & 0.198 & 0.202 & $0.199 \pm 0.007$ \\
\hline & 4 & 0.222 & 0.221 & $0.223 \pm 0.008$ \\
\hline & 5 & 0.246 & 0.243 & $0.241 \pm 0.008$ \\
\hline \multirow{5}{*}{26000} & 1 & 0.155 & 0.154 & $0.159 \pm 0.005$ \\
\hline & 2 & 0.177 & 0.179 & $0.181 \pm 0.006$ \\
\hline & 3 & 0.199 & 0.202 & $0.200 \pm 0.007$ \\
\hline & 4 & 0.223 & 0.222 & $0.221 \pm 0.007$ \\
\hline & 5 & 0.246 & 0.243 & $0.239 \pm 0.008$ \\
\hline \multirow{5}{*}{39200} & 1 & 0.155 & 0.154 & $0.158 \pm 0.005$ \\
\hline & 2 & 0.176 & 0.179 & $0.180 \pm 0.006$ \\
\hline & 3 & 0.200 & 0.202 & $0.201 \pm 0.006$ \\
\hline & 4 & 0.223 & 0.222 & $0.221 \pm 0.007$ \\
\hline & 5 & 0.246 & 0.243 & $0.240 \pm 0.007$ \\
\hline
\end{tabular}

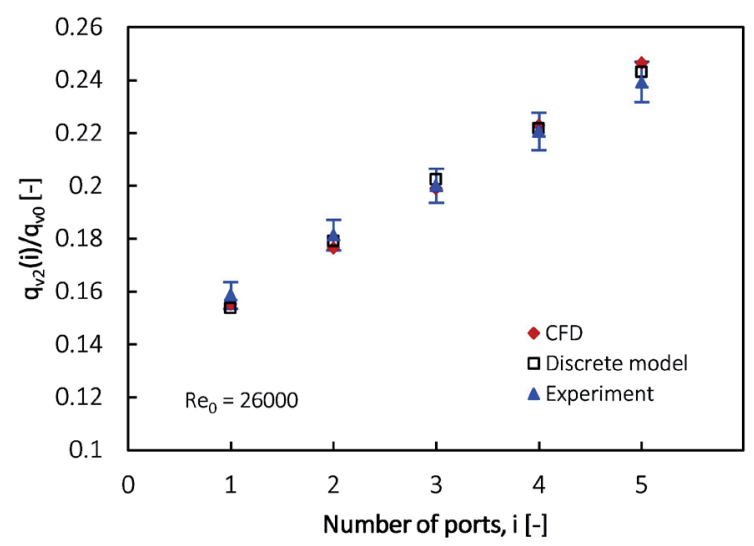

Fig. 8 Dimensionless volume flow rate distributions obtained by three different methods - representative results $\left(R e_{0}=26000\right)$

As can be seen from Table 2, fairly narrow experimental error bars are given for each of the measured dimensionless volume flow rates, and all the results of the three different methods are within these error bars. The measured distributions are accurately reproduced by the CFD and discrete models. In the investigated Reynolds number range, dimensionless volume flow rate distributions are almost independent of the Reynolds number at the manifold inlet. This observation is in accordance with the results of Kubo and Ueda [21]. The mechanism of the phenomenon is also discussed by Wang [23]. In short manifolds, where the ratio of manifold length to diameter is small, the effect of the momentum is dominated so that the effect of the friction can be neglected. In the present paper, a relatively short manifold is investigated; therefore, flow distributions depend slightly on the Reynolds number at the manifold inlet, as it was expected.

In order to validate the measured branched off volume flow rates at the three different Reynolds numbers, total volume flow rates are also determined by summing the volume flow rates through each of the ports measured by Pitot probe. Total volume flow rates measured by the through-flow orifice plate and deduced from velocity data are compared to each other, and good correspondence is found as can be seen in Table 3.

Table 3 Total volume flow rates measured by the through-flow orifice plate and deduced from velocity data

\begin{tabular}{lll}
\hline $\begin{array}{l}R e_{0}[-] \\
\text { Orifice plate }\end{array}$ & $\begin{array}{l}q_{v 0}\left[\mathrm{~m}^{3 / \mathrm{s}}\right] \\
\text { Orifice plate }\end{array}$ & $\begin{array}{l}q_{v 0}\left[\mathrm{~m}^{3} / \mathrm{s}\right] \\
\text { Pitot probe }\end{array}$ \\
\hline $13200 \pm 400$ & $0.0032 \pm 0.0001$ & $0.0034 \pm 0.0003$ \\
$26000 \pm 800$ & $0.0063 \pm 0.0002$ & $0.0067 \pm 0.0005$ \\
$39200 \pm 1200$ & $0.0095 \pm 0.0003$ & $0.0100 \pm 0.0007$ \\
\hline
\end{tabular}


Volume flow rates deduced from velocity data are slightly larger than those measured by the orifice meter. This overshoot ensues from the measurement technique: the applied resolution consisting of 21 measurement points, the distance between the measurement points and the outlet of ports and the directional sensitivity of the probe can increase the values of measured volume flow rates. However, all the differences between the results of the two different methods are smaller than the estimated uncertainties of total volume flow rates measured by Pitot probe.

Relative errors of total volume flow rates deduced from velocity data are larger than those of dimensionless volume flow rates; nevertheless, all the measurement accuracies reach the level of accuracy that is acceptable in engineering practice.

The agreement between dimensionless volume flow rate distributions determined by the three different methods is excellent. The presented CFD and discrete models are expansively applicable for design purposes; however, CFD is less suitable for geometry optimization due to the higher computational cost. Time-consuming experimental investigations with relatively expensive instrumentation are primarily applicable for validation and less appropriate for design tasks.

\section{Conclusions}

The applicability and accuracy of a new discrete dividingflow manifold model was investigated. The model used variable flow coefficients, which were computed according to the suggestions of former studies [4, 24]. A three-dimensional CFD model was also constructed, and dimensionless volume flow rate distributions along a specified dividing-flow manifold were calculated with the two theoretical models. Experimental investigations were performed, and results of calculations compared favourably with those of own experiments. The measured distributions were accurately reproduced by the CFD and discrete models.

The discrete model is flexible and expansively applicable for manifold design. CFD is less suitable for geometry optimization; however, the accuracies of our CFD results are adequate. Experimental investigation is the least flexible approach and unsuitable for design tasks; nevertheless, the available experimental database was extended, and these results can be used as reference data for further validation.

\section{Acknowledgement}

This paper relates to the scientific program of the project "Development of quality-oriented and harmonized $\mathrm{R}+\mathrm{D}+\mathrm{I}$ strategy and the functional model at BME", supported by the New Hungary Development Plan (Project ID: TÁMOP4.2.1/B-09/1/KMR-2010-0002). It is also supported by the project "Talent care and cultivation in the scientific workshops of BME” project (Project ID: TÁMOP-4.2.2/B-10/1-2010-0009).

\section{Nomenclature}

$\begin{array}{ll}A & \text { area [m] } \\ A_{1} & \text { constant } \\ A_{2} & \text { constant } \\ B_{1} & \text { constant } \\ B_{2} & \text { constant } \\ C & \text { constant } \\ C_{f} & \text { turning loss coefficient } \\ C_{f e} & \text { extended turning loss coefficient } \\ C V R & \text { critical velocity ratio } \\ D & \text { constant } \\ D_{1} & \text { inner diameter of header }[\mathrm{m}] \\ D_{2} & \text { inner diameter of ports }[\mathrm{m}] \\ i & \text { ith branch point } \\ j & \text { jth measurement point } \\ k & \text { pressure recovery factor } \\ L_{1} & \text { distance between two neighbouring laterals }[\mathrm{m}] \\ L_{2} & \text { length of ports [m] } \\ n & \text { total number of ports } \\ p & \text { static pressure }[\mathrm{Pa}] \\ p_{\mathrm{t}} & \text { total pressure [Pa] } \\ p_{0} & \text { ambient pressure [Pa] } \\ q_{v} & \text { volume flow rate [m } 3 / \mathrm{s}] \\ R e & \text { Reynolds number } \\ v & \text { flow velocity [m/s] } \\ \Delta P_{f} & \text { friction loss } \\ & \end{array}$

\section{Greek letters}

$\begin{array}{ll}\alpha & \text { constant for the calculation of } k \\ \beta & \text { constant for the calculation of } k \\ \lambda & \text { pipe friction factor } \\ \rho & \text { fluid density }\left[\mathrm{kg} / \mathrm{m}^{3}\right]\end{array}$

\section{Subscripts}

$\begin{array}{ll}l & \text { left control surface } \\ p & \text { belongs to Pitot probe measurements } \\ r & \text { right control surface } \\ 0 & \text { in the header before the first branch point } \\ 1 & \text { in the header } \\ 2 & \text { in the port }\end{array}$

\section{References}

[1] Holmes, J. D., Lewis, R. E. "Optimization of dynamic- pressure-measurement systems. II. Parallel tube-manifold systems." Journal of Wind Engineering and Industrial Aerodynamics. 25 (3). pp. 275-290. 1987. DOI: 10.1016/0167-6105(87)90022-5

[2] Liseth, P. "Wastewater disposal by submerged manifolds." Journal of the Hydraulics Division. 102 (1). pp. 1-14. 1976.

[3] Matsubara, Y. "Geometry design of a coat-hanger die with uniform flow rate and residence time across the die width." Polymer Engineering \& Science. 19 (3). pp. 169-172. 1979. DOI: 10.1002/pen.760190302 
[4] Wang, J., Gao, Z., Gan, G., Wu, D. "Analytical solution of flow coefficients for a uniformly distributed porous channel." Chemical Engineering Journal. 84 (1). pp. 1-6. 2001. DOI: 10.1016/S1385-8947(00)00263-1

[5] Wang, J. "Pressure drop and flow distribution in parallel- channel configurations of fuel cells: U-type arrangement." International Journal of Hydrogen Energy. 33 (21). pp. 6339-6350. 2008.

DOI: 10.1016/j.ijhydene.2008.08.020

[6] Wang, J. "Pressure drop and flow distribution in parallel- channel configurations of fuel cells: Z-type arrangement." International Journal of Hydrogen Energy. 35 (11). pp. 5498-5509. 2010. DOI: 10.1016/j.ijhydene.2010.02.131

[7] Bassiouny, M. K., Martin, H. "Flow distribution and pressure drop in plate heat exchangers-I U-type arrangement." Chemical Engineering Science. 39 (4). pp. 693-700. 1984. DOI: 10.1016/0009-2509(84)80176-1

[8] Hassan, J. M., Mohammed, W. S., Mohamed, T. A., Alawee, W. H. "Review on single-phase fluid flow distribution in manifold." International Journal of Science and Research (IJSR). 3 (1). pp. 325-330. 2014.

[9] Hassan, J. M., AbdulRazzaq, A., Kamil, B. K. "Flow distribution in manifolds." Journal of Engineering and Development. 12 (4). pp. 159-177. 2008.

[10] Hassan, J., M., Mohammed, W., S., Hameed, A. F. "Study of three dimensional fluid flow in manifold-laterals system." Engineering \& Technology Journal. 30 (7). pp. 1132-1148. 2012.

[11] Heggemann, M., Hirschberg, S., Spiegel, L., Bachmann, C. "CFD simulation and experimental validation of fluid flow in liquid distributors." Chemical Engineering Research and Design. 85 (1). pp. 59-64. 2007. DOI: $10.1205 /$ cherd06093

[12] Tonomura, O., Tanaka, S. Noda, M., Kano, M., Hasebe, S., Hashimoto, I. "CFD-based optimal design of manifold in plate-fin microdevices." Chemical Engineering Journal. 101 (1-3). pp. 397-402. 2004. DOI: $10.1016 /$ j.cej.2003.10.022

[13] Chen, A., Sparrow, E. M. "Turbulence modeling for flow in a distribution manifold." International Journal of Heat and Mass Transfer. 52 (5-6). pp. 1573-1581. 2009. DOI: 10.1016/j.ijheatmasstransfer.2008.08.006

[14] Kulkarni, A. V., Roy, S. S., Joshi, J. B. "Pressure and flow distribution in pipe and ring spargers: Experimental measurements and CFD simulation." Chemical Engineering Journal. 133 (1-3). pp. 173-186. 2007. DOI: 10.1016/j.cej.2007.03.011

[15] Kapadia, S., Anderson, W. K. "Sensitivity analysis for solid oxide fuel cells using a three-dimensional numerical model." Journal of Power Sources. 189 (2). pp. 1074-1082. 2009. DOI: 10.1016/j.jpowsour.2008.12.130

[16] Gandhi, M. S., Ganguli, A. A., Joshi, J. B., Vijayan, P. K. "CFD simulation for steam distribution in header and tube assemblies." Chemical Engineering Research and Design. 90 (4). pp. 487-506. 2012. DOI: 10.1016/j.cherd.2011.08.019
[17] Datta, A. B., Majumdar, A. K. "Flow distribution in parallel and reverse flow manifolds." International Journal of Heat and Fluid Flow. 2 (4). pp. 253-262. 1980. DOI: 10.1016/0142-727X(80)90019-3

[18] López, R., Lecuona, A., Ventas, R., Vereda, C. "A numerical procedure for flow distribution and pressure drops for $U$ and $\mathrm{Z}$ type configurations plate heat exchangers with variable coefficients." Journal of Physics: Conference Series. 395 (1). 2012. DOI: 10.1088/1742-6596/395/1/012060

[19] Wang, J., Wang, H. "Discrete method for design of flow distribution in manifolds." Applied Thermal Engineering. 89. pp. 927-945. 2015. DOI: 10.1016/j.applthermaleng.2015.06.069

[20] Acrivos, A., Babcock, B. D., Pigford, R. L. "Flow distributions in manifolds." Chemical Engineering Science. 10 (1-2). pp. 112-124. 1958. DOI: 10.1016/0009-2509(59)80030-0

[21] Kubo, T., Ueda, T. "On the characteristics of divided flow and confluent flow in headers." Bulletin of JSME. 12 (52). pp. 802-809. 1969.

[22] Bajura, R. A. "A model for flow distribution in manifolds." ASME Journal of Engineering for Power. 93 (1). pp. 7-12. 1971.

DOI: $10.1115 / 1.3445410$

[23] Wang, J. "Theory of flow distribution in manifolds." Chemical Engineering Journal. 168 (3). pp. 1331-1345. 2011.

DOI: 10.1016/j.cej.2011.02.050

[24] Kristóf, G., Tomor, A. "Loss coefficient of finite length dividing junctions." In: Proceedings of Conference on Modelling Fluid Flow (CMFF'15). The 16th International Conference on Fluid Flow Technologies. Budapest, Hungary, September 1-4, 2015.

[25] Pigford, R. L., Ashraf, M., Miron, Y. D. "Flow distribution in piping manifolds." Industrial \& Engineering Chemistry Fundamentals. 22 (4). pp. 463-471. 1983. DOI: 10.1021/i100012a019

[26] Menter, F. R. "Two-equation eddy-viscosity turbulence models for engineering applications." AIAA Journal. 32 (8). pp. 1598-1605. 1994. DOI: $10.2514 / 3.12149$

[27] Celik, I. B., Ghia, U., Roache, P. J., Freitas, C. J. "Procedure for estimation and reporting of uncertainty due to discretization in CFD applications." ASME Journal of Fluids Engineering. 130 (7). 078001. 2008. DOI: $10.1115 / 1.2960953$

[28] Standard ISO 5167-2:2003. "Measurement of fluid flow by means of pressure differential devices inserted in circular cross-section conduits running full - Part 2: Orifice plates." 2003

[29] Measurement of fluid flow in closed conduits. Velocity area method using Pitot static tubes. ISO 3966-1977 standard. 1977. (Hungarian standard: MSZ 1709/17-84)

[30] Gupta, S. V. "Measurement uncertainties: Physical parameters and calibration of instruments." Chapter 5. pp. 109-129. Springer, Berlin, Heidelberg. 2012. DOI: 10.1007/978-3-642-20989-5 